\title{
Brandversuch mit E-Fahrzeugen in Tunnelanlagen
}

\author{
Peter Sturm ${ }^{1}$, Patrik Fössleitner², Daniel Fruhwirt', Andrea Schirmer ${ }^{1}$, Bernhard Reinwald ${ }^{3}$, Manuel Kühbacher ${ }^{3}$, \\ Robert Wenighofer ${ }^{3}$, Simon Heindl ${ }^{4}$, Thomas Nöst ${ }^{1}$, Philip Leonhardt ${ }^{1}$, Alexander Hödl ${ }^{4}$ und Stefan Krausbar ${ }^{5}$
}

${ }^{1}$ Institut für Verbrennungskraftmaschinen und Thermodynamik, Technische Universität Graz, Graz, Österreich ${ }^{2}$ FVT mbH, Graz, Österreich

${ }^{3}$ Lehrstuhl für Subsurface Engineering, Montanuniversität Leoben, Leoben, Österreich

${ }^{4}$ Institut für Fahrzeugsicherheit, TU Graz, Graz, Österreich

${ }^{5}$ Österreichischer Bundesfeuerwehrverband, Wien, Österreich

Eingegangen 24. Oktober 2020; angenommen 27. Oktober 2020; online publiziert 19. November 2020

Zusammenfassung: Im Rahmen des Forschungsprojektes BRAFA werden die Auswirkungen von Bränden von E-Fahrzeugen auf die Sicherheit von Tunnelbenutzern und die Tunnelinfrastruktur untersucht sowie Methoden zur Bekämpfung von Bränden von E-Fahrzeugen bewertet. Basis dazu sind Realbrandversuche, die im "Zentrum am Berg" in geeigneten Versuchstunneln durchgeführt werden. Auf Basis der bis jetzt durchgeführten Realbrandversuche an Batterien und Gesamtfahrzeugen kann geschlossen werden, dass wichtige Parameter wie die Wärmefreisetzungsrate und die zu erwartenden Temperaturen bei E-Fahrzeugbränden im PKW Bereich ähnlich jener konventionell angetriebener Fahrzeuge sind. Problematisch erscheint jedoch die erhöhte Freisetzung von schädlichen Gasen wie HF und Phosphorsäure sowie die Tatsache, dass Löschversuche mit „üblichen“ Wassermengen nicht zielführend sind. Hier zeigt sich, dass eine effektive Einbringung von Wasser in den unmittelbaren Batteriebereich sehr günstige Auswirkungen auf die Verhinderung/Unterdrückung der thermischen Fortpflanzung des Brandes hat. Im noch verbleibenden Zeitraum des Forschungsprojektes steht eine detaillierte Datenanalyse sowie eine Hochrechnung auf Brandereignisse größerer E-Fahrzeuge (z.B. Busse) sowie die Einbindung der Ergebnisse in die Tunnelrisikoanalysemodelle an.

Schlüsselwörter: E-Fahrzeuge, Brandversuche,

Straßentunnel
Full Scale Fire Tests with Batterie Electric Vehicles in Road Tunnels

Abstract: In the framework of the BRAFA research project, the effects of e-vehicle fires on the safety of tunnel users and the tunnel infrastructure are examined, and methods for fighting fires are evaluated. The basis for this are full-scale fire tests carried out at the tunnel research centre "Zentrum am Berg" in Austria. First results based on the fullscale fire tests of battery packs and complete vehicles, it can be concluded that important parameters such as the heat release rate and the temperatures of such fires are similar to those of conventionally powered vehicles. However, the increased release of harmful gases such as hydrogen fluoride and phosphoric acid are considered critical as well as the fact that attempts to extinguish the fire with 'typical' amounts of water do not lead to the desired results. This shows that an effective injection of water into the battery casing has very beneficial effects on preventing/suppressing the thermal propagation of the fire. In the remaining period of the research project, a detailed data analysis as well as an extrapolation of the results to fire incidents in larger electric vehicles (e.g. buses) and the integration of the results into the tunnel risk analysis models are due.

Keywords: E-mobility, Battery electric vehicles, Full scale fire tests, Road tunnels

\section{Einleitung}

Die E-Mobilität wird derzeit als ein probates Mittel zur Reduktion der Treibhausgasemissionen des Sektors Verkehr angesehen. Dies spiegelt sich in der in den letzten Jahren merklich gestiegenen Anzahl von Fahrzeugen mit batterieelektrischem Antrieb im Straßenverkehr. Obwohl derartige Antriebssysteme vornehmlich in Personenkraftwagen

\footnotetext{
Ao. Univ.-Prof. Dipl.-Ing. Dr. techn. P. Sturm ( $\varangle)$ Institut für Verbrennungskraftmaschinen und Thermodynamik Technische Universität Graz, Inffeldgasse 25c, $8010 \mathrm{Graz}$, Österreich sturm@ivt.tugraz.at
} 


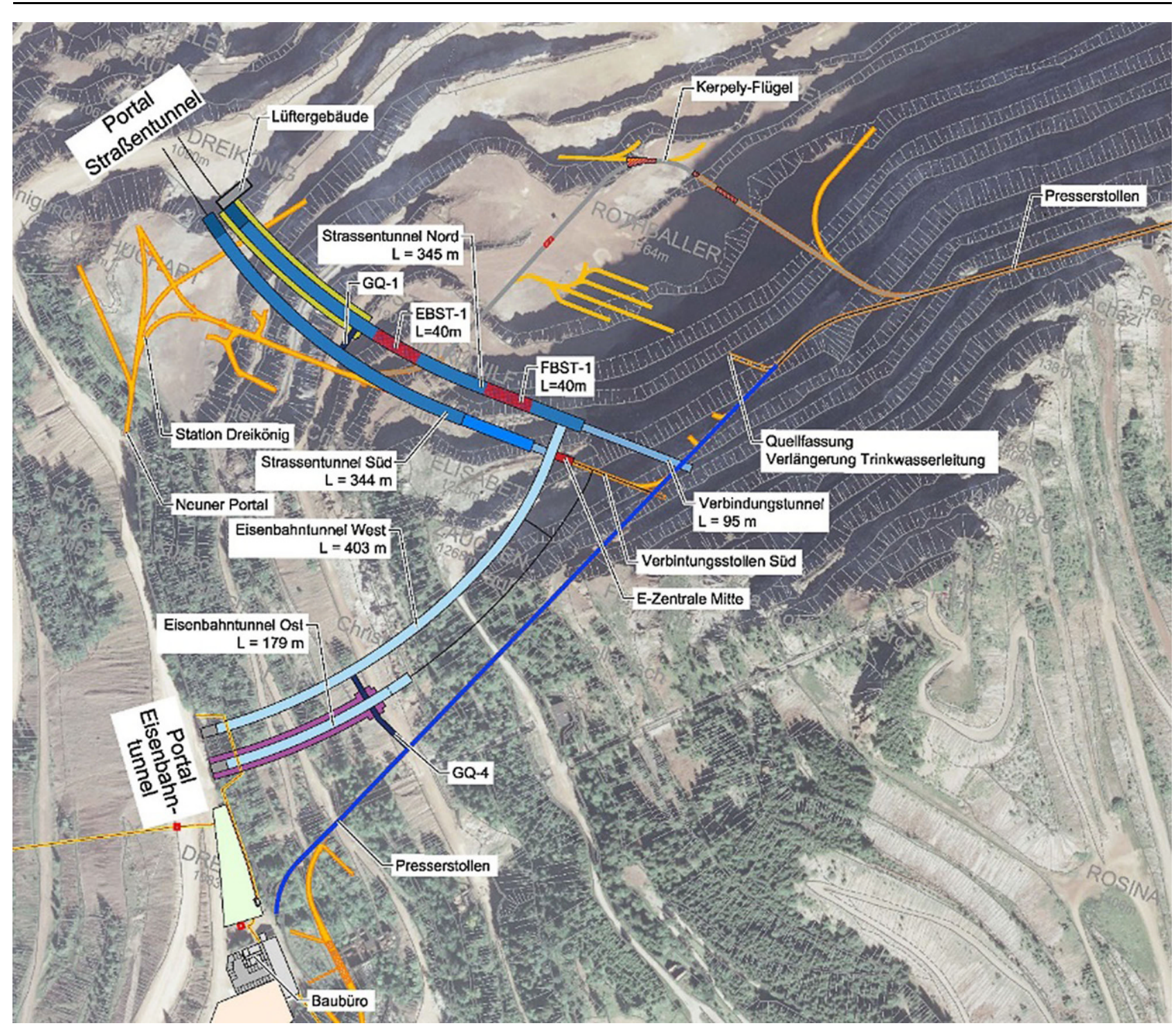

Abb. 1: Grundriss der Untertage-Anlagen im „Zentrum am Berg“

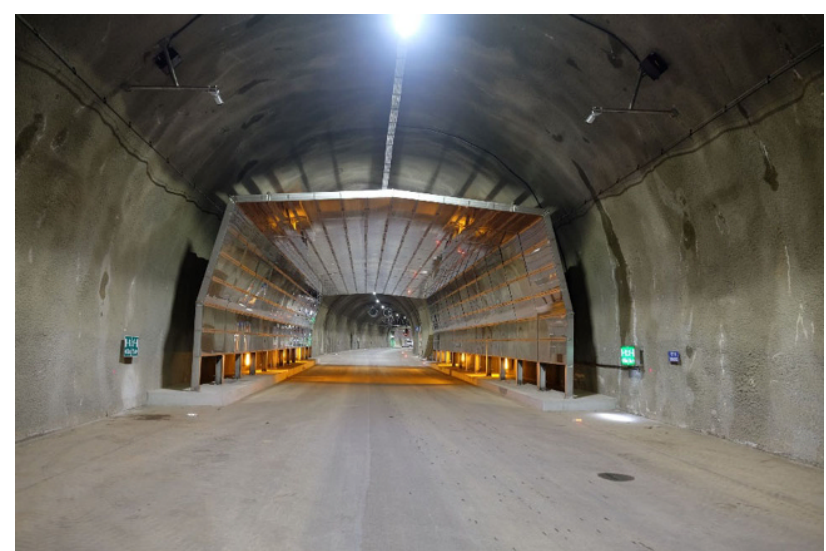

Abb. 2: Versuchsstollen, Tunnelabschnitt mit Hitzeschild für Brandversuche mit hohen Brandlasten mit einem noch eingeschränkten Wirkungsbereich zu finden sind, geht die Entwicklung in Richtung Batteriesysteme mit höheren Leistungsdichten, um einerseits die Fahrleistungen zwischen den Ladevorgängen zu erhöhen und andererseits auch in den Nutzfahrzeugverkehr vorzustoßen.

Die derzeitigen Batteriegenerationen bauen stark auf eine Lithium-Ionen Technologie auf, die im Falle eines Brandereignisses - wodurch auch immer dieses zustande kommen mag - ein gänzlich anderes Verhalten als herkömmliche Energieträger (flüssige Kraftstoffe) aufweist. Dies bezieht sich auf die Brandursache ebenso wie auf Brandverlauf und Gefahrenrisiko durch die entstehenden Rauchgase. Besonders kritisch ist dies in unterirdischen Verkehrsanlagen oder im Garagenbereich, da hier die Brandbekämpfung erschwert ist und die Verdünnungsmöglichkeiten giftiger Rauchgase stark eingeschränkt sind.

Um diesen Problemen verstärkt auf wissenschaftlicher Basis auf den Grund gehen zu können, finden auf internationaler Ebene in bescheidenem Maße Untersuchungen zum 

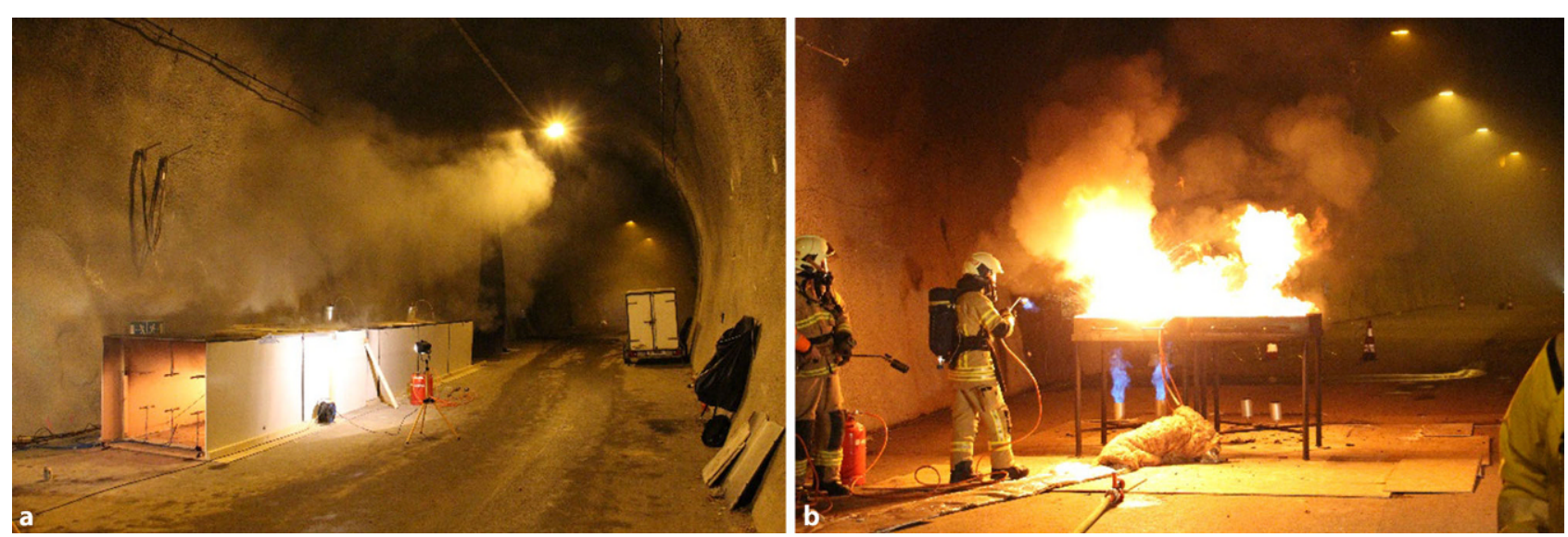

Abb. 3: a,b Versuchsaufbau für Einzelmodule

Pack-sized Concentration Gasanalyzer Test $\# 8$

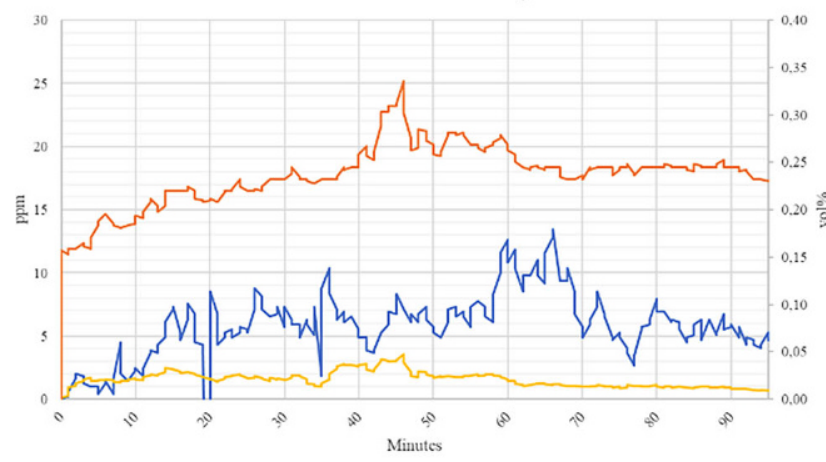

$-\mathrm{CO}[\mathrm{ppm}]-\mathrm{NOx}[\mathrm{ppm}]-\operatorname{CO2}[\mathrm{val} \%]$

Pack-sized Concentration QAT Stacks + Filter Test \#8

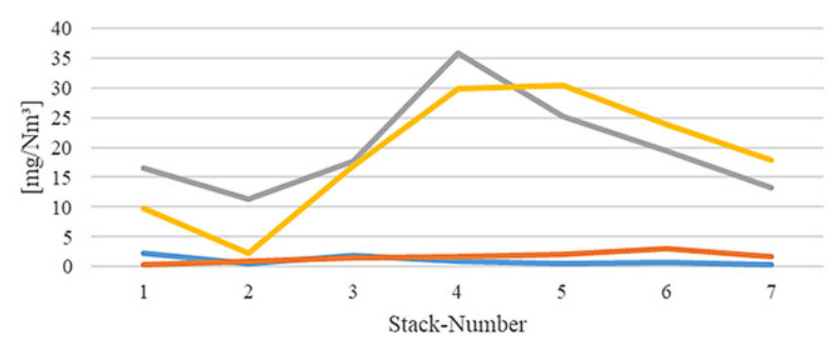

$\longrightarrow \mathrm{HCl}+\mathrm{Cl} \longrightarrow \mathrm{SO} 2+\mathrm{SO} 4-\mathrm{H} 3 \mathrm{PO} 4+\mathrm{PO} 4-\mathrm{HF}+\mathrm{F}$
Pack-sized Concentration Gasanalyzer Test \#9

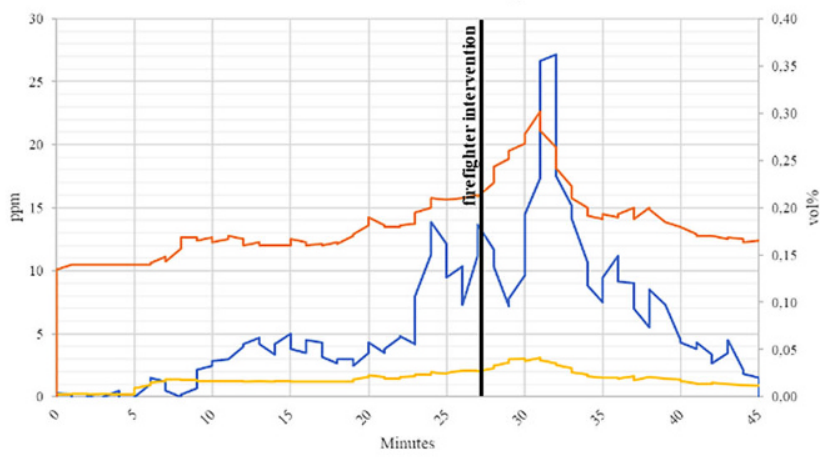

$-\mathrm{CO}[\mathrm{ppm}]-\mathrm{NOr}[\mathrm{ppxn}]-\mathrm{CO}[\mathrm{vol} \%]$

Pack-sized Concentration QAT Stacks + Filter Test \#9

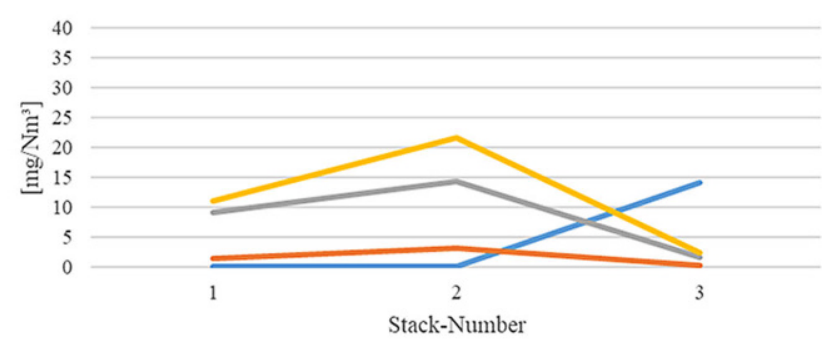

$\longrightarrow \mathrm{HCl}+\mathrm{Cl} \longrightarrow \mathrm{SO} 2+\mathrm{SO} 4-\mathrm{H} 3 \mathrm{PO} 4+\mathrm{PO} 4-\mathrm{HF}+\mathrm{F}$

Abb. 4: Konzentrationsverläufe der Versuche \#8 und \#9
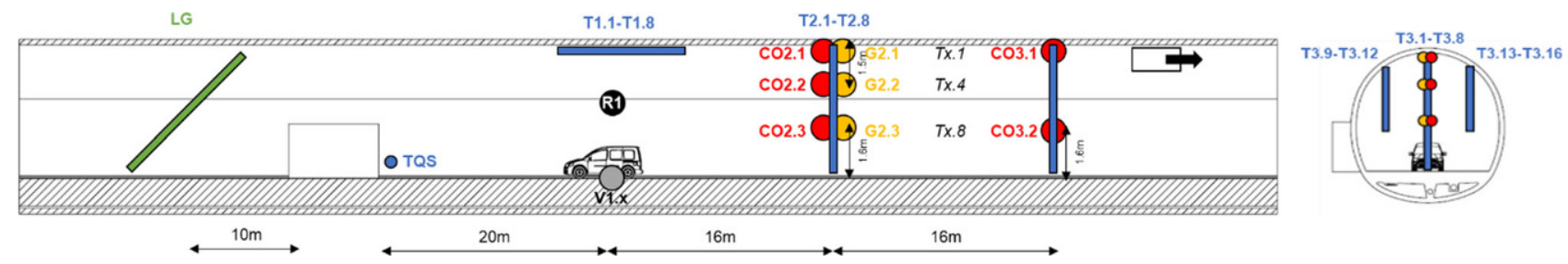

Abb. 5: Messaufbau 


\begin{tabular}{|lllllll}
\hline $\begin{array}{l}\text { TABELLE } 1 \\
\text { Hauptparameter der Versuche }\end{array}$ & & & \\
\hline Versuch \# & Zelltechnologie & Zelltyp & Kapazität/Zelle [Ah] & $\begin{array}{l}\text { Anzahl Zellen pro } \\
\text { Modul }\end{array}$ & $\begin{array}{l}\text { Anzahl der } \\
\text { Module }\end{array}$ & SOC [\%] \\
\hline 8 & LFP & Prismatisch & 20 & 40 & 18 & Unknown \\
\hline 9 & NMC & Prismatisch & 60 & 13 & 27 & 100 \\
\hline LFP Lithium & Eisen Phosphat, NMC Lithium lonen (Lithium-Nickel-Mangan-Cobalt-Oxide) & & \\
\hline
\end{tabular}

Brandverhalten von batteriebetriebenen Fahrzeugen - ausschließlich in der PKW Klasse - statt. In Österreich wurde im Zuge der Verkehrsinfrastrukturforschung (VIF) 2018 ein Projekt zur Erweiterung des Wissensstandes in diesem Bereich initiiert. Unter Federführung der TU Graz arbeiten die MU Leoben, der Österreichische Bundesfeuerwehrverband sowie die ILF Beratende Ingenieure im Projekt BRAFA zusammen, um das Brandverhalten derartiger Fahrzeuge und dessen Auswirkungen auf die Sicherheit von Tunnelnutzern zu untersuchen (BRAFA, 1). Kernpunkt dieser Untersuchungen sind Realbrandversuche in Tunnelanlagen, um unter realistischen Randbedingungen die Brandentwicklung und die daraus resultierenden Folgen studieren zu können. Während die Messungen der Brandparameter einen Teil der Versuche ausfüllten, war ein zweiter den Möglichkeiten zur Löschung bzw. Eindämmung des Brandes gewidmet. Dieser zweite Teil diente vor allem dazu, qualitative Aussagen über die Einsatztaktik der Feuerwehr zu tätigen.

Die Versuche wurden allesamt im neu errichteten Tunnelforschungszentrum "Zentrum am Berg" in Eisenerz im Zeitraum 2019/2020 durchgeführt (ZAB, 2). Derzeit laufen die Detailanalysen der Versuche und die Beurteilung der Auswirkungen. Das Forschungsprogramm soll im Sommer 2021 abgeschlossen werden.

\section{Ausgangslage}

Bisherige Untersuchungen haben gezeigt, dass es bei Unfällen mit E-Fahrzeugen zwei Problemkreise gibt, die (vor allem im Nutzfahrzeugbereich) noch gänzlich unerforscht sind:

- Schädigungen einzelner Zellen führen zum sogenannten "thermal runaway", der in weiterer Folge zu einer massiven Überhitzung der Batterie und zu einem Austritt von kritischen hochgiftigen und brennbaren Substanzen führt.

- Brandverhalten und Schadstoffbildung, wenn ein Brandübergriff auf eine Batterie erfolgt.

Der derzeitige Stand des Wissens bezieht sich auf die Erfassung der Auswirkungen von Bränden einzelner Batteriezellen und kleineren Akkupacks [3] sowie Abschätzungen und Einzelversuche für PKW [4]. Dabei zeigt sich, dass unter anderem Fluorwasserstoff (Flusssäure, HF) entstehen kann, der als hochtoxisch einzustufen ist und für Ersthelfer bzw. Einsatzkräfte ein unbekanntes Risiko darstellt [5]. Herkömmliche Brandbekämpfungsmaßnahmen erscheinen nicht zielführend, da derartige Batterien genügend Eigen- energie und Brennstoffe haben, um Wiederentzündungen zu verursachen.

Anmerkung: Obwohl es sich beim Energiespeicher um Akkumulatoren handelt, ist auch im deutschen Sprachgebrauch in der Fahrzeuganwendung das Wort Batterie gebräuchlich. Daher wird auch hier in weiterer Folge von (wiederaufladbaren) Batterien gesprochen.

\section{Projektdurchführung}

\subsection{Versuchsaufbau}

Kernaktivität des Projektes ist die Durchführung von Realbrandversuchen in einer Tunnelanlage. Mit dem zur Verfügung stehenden „Zentrum am Berg“, einer Außenstelle der Montanuniversität Leoben, steht ein weltweit einzigartiges Forschungszentrum zur Verfügung, das derartige Versuche in realen Tunnelanlagen ermöglicht. Mit einer Gesamtlänge von ca. $2 \mathrm{~km}$ umfasst es Tunnelbereiche mit Straßentunnelquerschnitten im Endausbau und Rohbau genauso wie Tunnelquerschnitte, die den gängigen einröhrigen Eisenbahntunneln entsprechen. Alle diese Teilbereiche sind fast unabhängig voneinander betreibbar. Abb. 1 zeigt eine Grundrissdarstellung des Tunnelkomplexes, Abb. 2 einen Teilabschnitt des Straßentunnels, der speziell für Brandversuche mit höheren Brandlasten ausgestattet ist. Die Realbrandversuche für dieses Projekt wurden in einem Tunnelabschnitt im Rohbauzustand durchgeführt, da dieser Bereich die für den damaligen Zeitpunkt günstigsten Rahmenbedingungen hinsichtlich Belüftungsmöglichkeiten, Fluchtwegsituierung und Infrastruktur für Messtechnik aufwies.

Als Messgrößen dienten in erster Linie Temperaturverteilungen im Tunnelquerschnitt, Temperaturbelastungen der Tunnelwände, die Konzentrationen der Rauchgase sowie bei durchgeführten Löschversuchen auch die Analyse des Löschwassers. Seitens der Batteriemodule erfolgte eine Temperatur- und Spannungsüberwachung einzelner Zellen. Für die Gasmessungen wurden Filter-Stack-Messungen für die Komponenten $\mathrm{HF}, \mathrm{HCl}, \mathrm{SO}_{2}$ und $\mathrm{H}_{3} \mathrm{PO}_{4}$ und kontinuierliche Messverfahren für die Stickoxide sowie für $\mathrm{CO}, \mathrm{CO}_{2}$ und $\mathrm{O}_{2}$ eingesetzt.

\subsection{Versuchsdurchführung}

Die Versuchsdurchführung erfolgte gestaffelt, beginnend bei einzelnen Batteriemodulversuchen bis hin zu Gesamtfahrzeugen. Prinzipiell wurde versucht, die Luftströmung im Tunnel mittels mechanischer Ventilation auf einen Bereich um 1,5 bis $2 \mathrm{~m} / \mathrm{s}$ einzustellen. Dies entspricht dem 

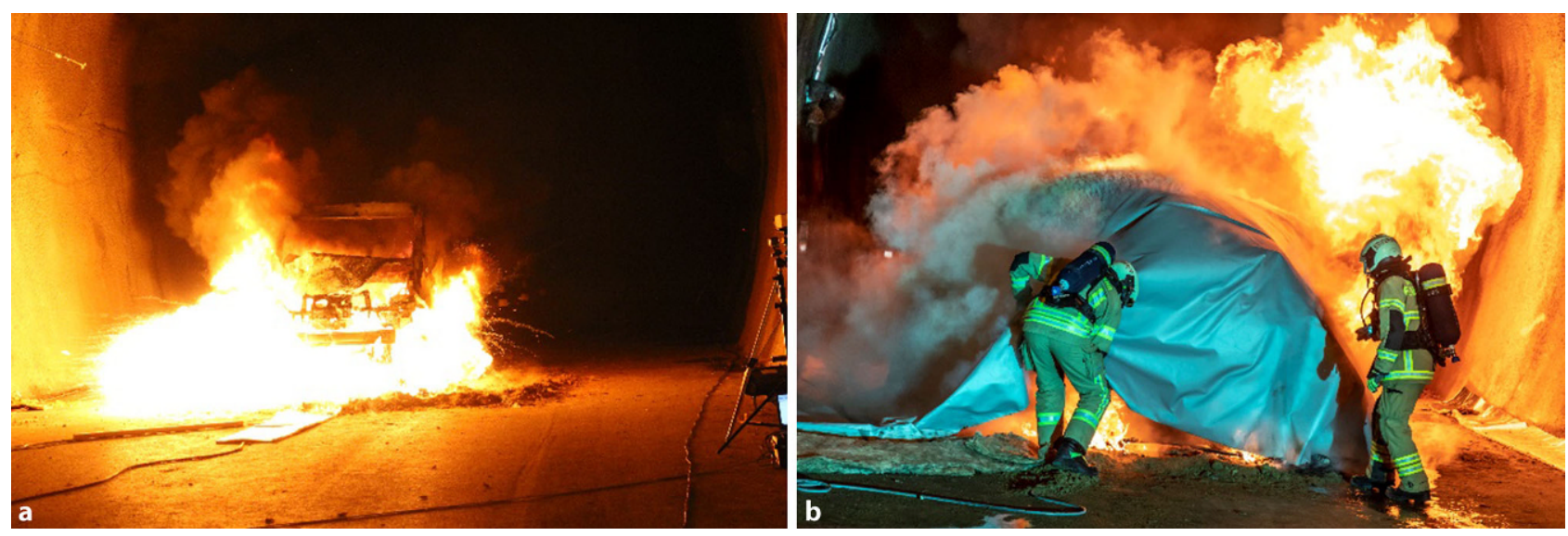

Abb. 6: Brandversuch E-Fahrzeug Lieferwagen (a, ( IVT), Löschversuche mit Branddecke (b, ( TU Graz-Lunghammer)

Regelbereich der Luftströmung in einem österreichischen Tunnel im Brandfall.

\subsubsection{Modulversuche}

Die erste Versuchsserie betraf Batteriemodule mit unterschiedlicher Zellchemie und in verschiedener Anzahl. Um eine verlässliche Messung der Gaskonzentrationen bewerkstelligen zu können, wurde ein eigener Versuchsaufbau mit einem verkleinerten Querschnitt verwirklicht (siehe Abb. 3a). Eine zweite Versuchsserie erfolgte mit einer Anzahl von Batteriemodulen, wie sie der Anordnung in einem handelsüblichen E-Fahrzeug entspricht. Diese Module wurden in Brandtassen im freien Tunnelquerschnitt angeordnet (siehe Abb. 3b). Bei allen Versuchen waren die einzelnen Module physisch nebeneinander angeordnet, jedoch nicht elektrisch verbunden. Zudem lag ein Ladungsgrad von $100 \%$ vor.

In Summe wurden mit diesen beiden Testaufbauten 9 Versuche durchgeführt. Exemplarisch sind in Abb. 4 die Konzentrationsverläufe der Versuche 8 und 9 dargestellt. Beide Versuche wurden im vollen Tunnelquerschnitt durchgeführt. Die wichtigsten Parameter der Module finden sich in Tab. 1, ermittelte Konzentrationsverläufe in Abb. 4.

Betrachtet man die Ergebnisse der indikativen Messungen, so zeigt sich, dass bei den Versuchen mit den Batterien die HF-Konzentrationen im Abgas in beachtenswerten Höhen liegen und mit bisher bekannten Untersuchungsergebnissen gut übereinstimmen [6].

\subsubsection{Fahrzeugversuche}

Versuche mit Gesamtfahrzeugen sind rar und in der publizierten Literatur auf PKW beschränkt. Im Rahmen diese Forschungsprojektes wurden Brandversuche an Gesamtfahrzeugen durchgeführt, wobei immer versucht wurde, ein batterieelektrisch betriebenes Fahrzeug einem baugleichen konventionell betriebenen Fahrzeug gegenüberzustellen. Da die durchgeführten Versuche auch zur Erprobung unterschiedlicher Einsatztaktiken zur Brandbekämpfung dienten, ist der Datenumfang bezüglich Brandverhalten und ungestörter Rauchgasentwicklung eingeschränkt. Dies ist jedoch den limitierten finanziellen Mitteln für die Versuchsdurchführung geschuldet.

Der Messaufbau (siehe Abb. 5) umfasste Messungen des Temperaturprofiles $(T)$ und der Gaskonzentrationen (GC), über die Höhe des Tunnels in verschiedenen Abständen vom Brandort, der Längsgeschwindigkeit der Tunnelluft (LG) stromaufwärts des Brandes sowie der Strahlung (MR) und der Batteriespannung (MV). Abb. 6a zeigt die Brandentwicklung eines BEV, bei dem der Batterieteil bereits am Brandgeschehen voll beteiligt ist. Im rechten Teil des Bildes ist der Einsatz einer Löschdecke zur vorübergehenden Brandeindämmung bzw. Verhinderung der Ausbreitung des Brandes dargestellt.

Abb. 7 zeigt exemplarisch Auswertungen der Temperaturverteilung und Gaskonzentrationen während des Brandes eines E-Fahrzeuges. Wie ersichtlich, liegen die Temperaturen der Rauchgase im Überkopf-Bereich bei 75 bis $80^{\circ} \mathrm{C}$ und stellen somit - wie bei einem PKW Brand zu erwarten - kein besonderes Sicherheitsrisiko dar. Die CO-Konzentrationen im oberen Tunnelbereich um 200 ppm und im unteren Bereich um 50 ppm (mit Spitzen bis 130 ppm) stellen ebenfalls kein unmittelbares Risiko dar. Die Wärmefreisetzungsrate weist einen Spitzenwert um $8 \mathrm{MW}$ und eine durchschnittliche Wärmefreisetzungsrate um $4 \mathrm{MW}$ auf. Bei diesen Parametern ist kein relevanter Unterschied zu einem Brand eines konventionellen Fahrzeuges feststellbar. Anzumerken ist jedoch, dass die Auswertungen zu den kritischen Rauchgasen $\mathrm{HF}, \mathrm{HCl}$ und Phosphorsäure noch ausständig sind.

Wie bereits angeführt, ist es auch Ziel des Projektes, Strategien zur Bekämpfung von Bränden durch die Einsatzkräfte zu untersuchen. Abb. 6b zeigt den Einsatz einer Löschdecke zur Reduktion des Brandgeschehens. Im Vergleich zu konventionellen Fahrzeugbränden enthält der Energiespeicher (Li-lonen Batterie) Bestandteile, die Sauerstoff enthalten. D.h. eine Reduktion des Brandgeschehens durch Sauerstoffentzug ist nur eingeschränkt möglich. Die durch den Bundesfeuerwehrverband durchgeführten diesbezüglichen Versuche demonstrierten die eingeschränkten Möglichkeiten des Löschens eines E-Fahrzeuges. Es zeigte sich jedoch, dass vor allem die gezielte Kühlung des Energie- 
Abb. 7: BEV Brandversuch: Temperaturverteilungen und Gaskonzentrationen (a), Wärmefreisetzungsrate (b)

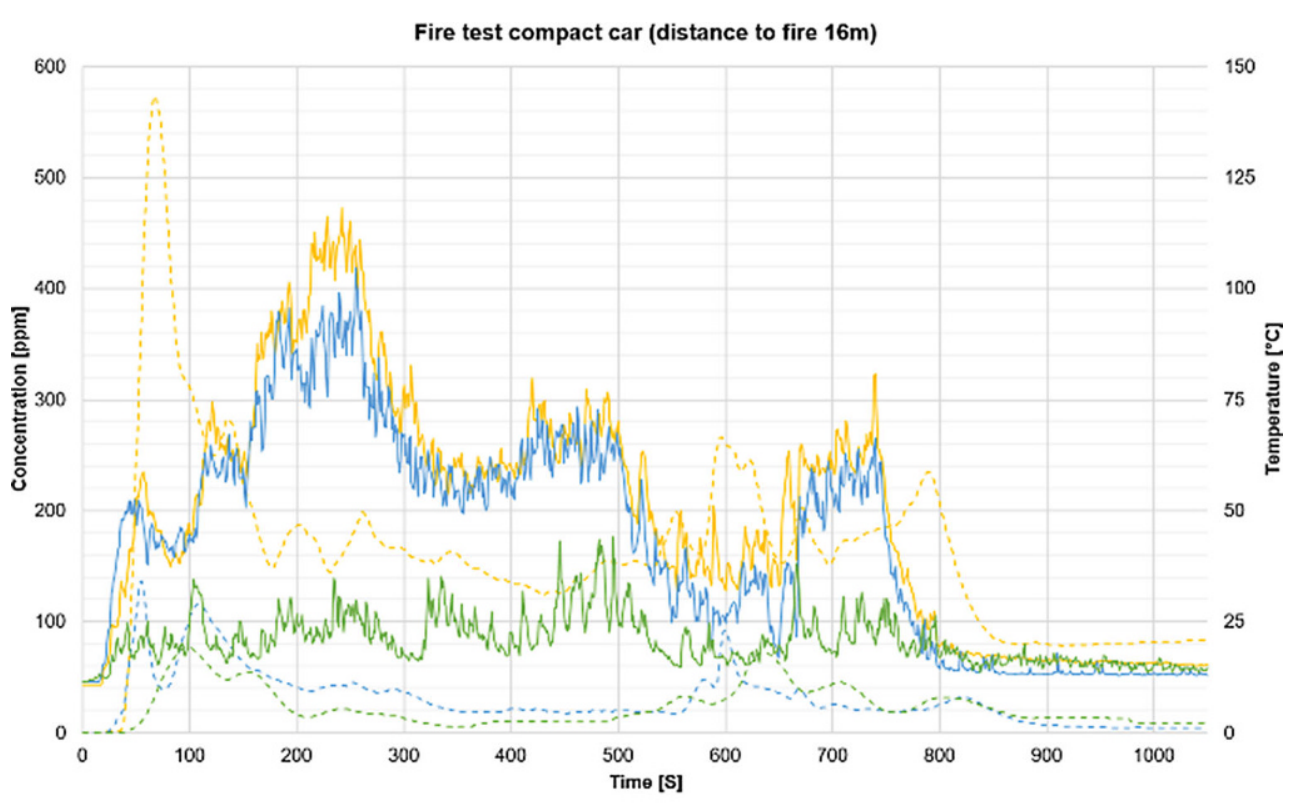

a

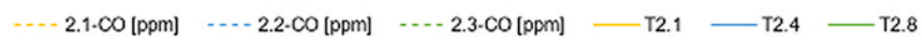

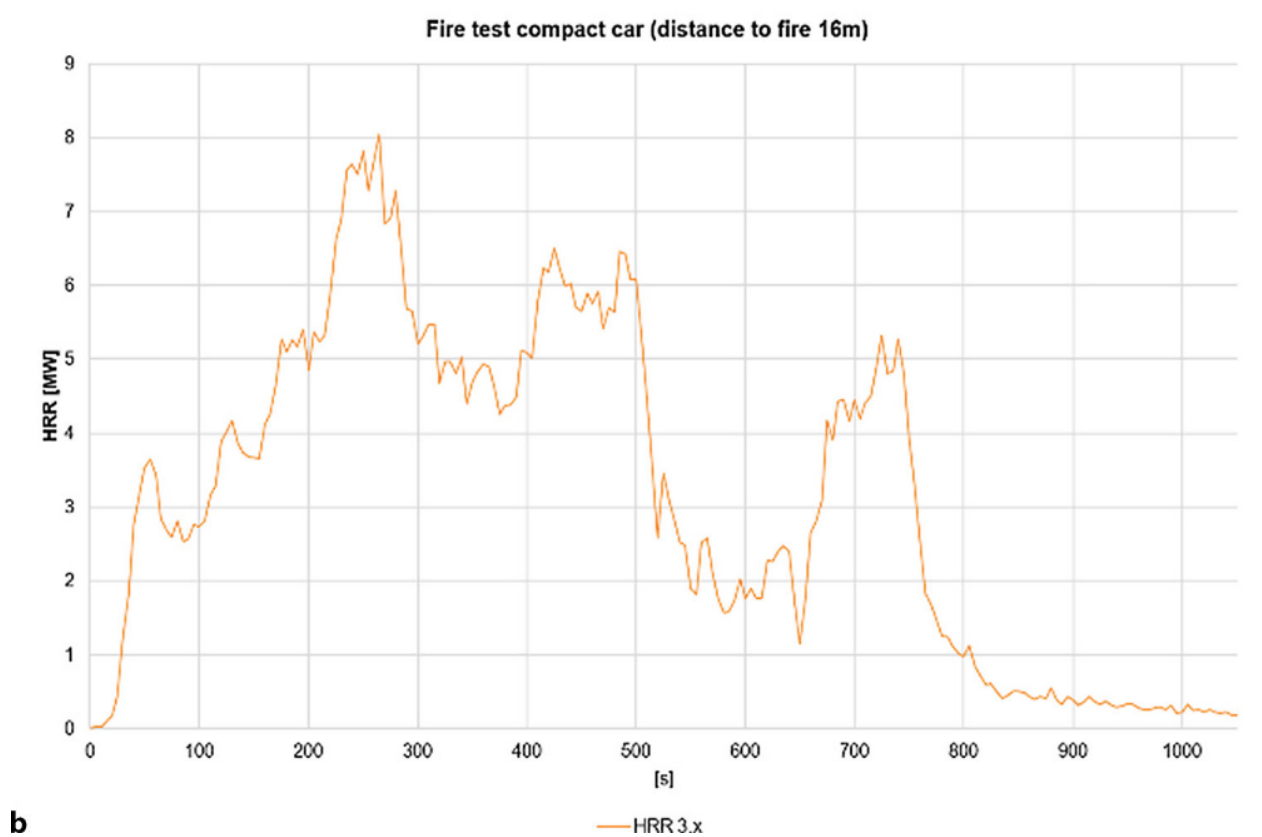

speichers eine effiziente Methode zur Reduktion der Brandlast darstellen würde.

\section{Zusammenfassung}

Während Brandereignisse konventioneller Fahrzeuge hinlänglich in ihrer Auswirkung auf die Sicherheit von Tunnelnutzern und das Bauwerk erforscht sind, ist dies bei E-Fahrzeugen nicht der Fall. Im Rahmen des VIF Forschungsprogrammes wurde daher ein Projekt zur Untersuchung des Brandverhaltens von E-Fahrzeugen in Tunnelanlagen initiiert. Kern dieses Projektes sind am "Zentrum am Berg" durchgeführte Realbrandversuche an E-Fahrzeugen und vergleichbaren Fahrzeugen mit konventionellen Antrieben. Diese Versuche haben zum Inhalt, das Brandverhalten und die Auswirkungen der Brände zu untersuchen und unterschiedliche Löschstrategien für E-Fahrzeug-Brände zu bewerten. Bis dato fanden mehrere Realbrandversuche an PKW statt, die zeigten, dass das Brandverhalten von E-Fahrzeugen sich nicht wesentlich von jenem konventioneller Fahrzeuge unterscheidet. Merklich unterschiedlich ist jedoch die Rauchgaszusammensetzung durch das vermehrte Auftreten von Fluorwasserstoff und Phosphorsäure, die beide ein erhöhtes Gesundheitsrisiko darstellen. Moderne Hochleistungsbatterien auf Li-Ionen Basis haben 
den im Brandfall notwendigen Sauerstoff in sich, sodass herkömmliche Löschstrategien mit Wassereinsatz nicht ausreichen. Es bedarf vielmehr einer effizienten Kühlung der Energiespeicher, um die thermische Fortpflanzung des Brandes zu unterbinden. Die bisher durchgeführten Versuche zeigten, dass hier die unmittelbare Einbringung von Wasser in den Batteriebereich ein Weg sein könnte, auch Batteriebrände in ihrer Entwicklung zu hemmen. Im noch zur Verfügung stehenden letzten Jahr des Forschungsprojektes liegt nun der Fokus auf einer detaillierten Datenanalyse, einer Hochrechnung der Auswirkungen auf leistungsstärkere Fahrzeugkategorien wie E-Busse und einer Einbindung der Ergebnisse in die Methoden zur Risikobewertung für Straßentunnel [7].

Förderung. Das Forschungsprojekt BRAFA wird unter der Projektnummer 873151 von der Österreichischen Forschungsförderungsgesellschaft mbH (FFG) im Auftrag des Bundes sowie der ASFINAG finanziert. Die beschriebenen Tätigkeiten erfolgten unter Mitarbeit der Projektpartner Montanuniversität Leoben, Lehrstuhl für Subsurface Engineering (Univ.-Prof. Dr. Robert Galler), TU Graz, Institut für Fahrzeugsicherheit (interne Projektleitung Assoc.-Prof. Dr. Wolfgang Sinz), Österreichischer Bundesfeuerwehrverband, ILF Consulting Engineers Austria mbH (Dr. Bernhard Kohl, Mag. Oliver Heger).

Funding. Open access funding provided by Graz University of Technology.

Open Access Dieser Artikel wird unter der Creative Commons Namens nennung 4.0 International Lizenz veröffentlicht, welche die Nutzung, Vervielfältigung, Bearbeitung, Verbreitung und Wiedergabe in jeglichem Medium und Format erlaubt, sofern Sie den/die ursprünglichen Autor(en) und die Quelle ordnungsgemäß nennen, einen Link zur Creative Commons Lizenz beifügen und angeben, ob Änderungen vorgenommen wurden.
Die in diesem Artikel enthaltenen Bilder und sonstiges Drittmaterial unterliegen ebenfalls der genannten Creative Commons Lizenz, sofern sich aus der Abbildungslegende nichts anderes ergibt. Sofern das betreffende Material nicht unter der genannten Creative Commons Lizenz steht und die betreffende Handlung nicht nach gesetzlichen Vorschriften erlaubt ist, ist für die oben aufgeführten Weiterverwendungen des Materials die Einwilligung des jeweiligen Rechteinhabers einzuholen.

Weitere Details zur Lizenz entnehmen Sie bitte der Lizenzinformation auf http://creativecommons.org/licenses/by/4.0/deed.de.

\section{Literatur}

1. BRAFA (2020). https://projekte.ffg.at/projekte/3290205. Zugegriffen: 23.10.2020

2. ZaB (2020). https://www.zab.at/. Zugegriffen: 23.10 .2020

3. Feng, X. O. (vol. 10 2018). Thermal runaway mechanism of lithium ion battery for electric vehicles: A review. Energy Storage Materials, S. $246-267$

4. Lam, C. M. (2016). Full-Scale Fire Testing of Electric and Internal Combustion Engine Vehicles. In Proceedings from 4th International Conference on Fires in Vehicles -FIVE 2016

5. Truchot, B. F. (No 97 2018). An experimental evaluation of toxic gas emissions from vehicle fires. Fire Safety Journal, S. 111-118. https:// doi.org/10.1016/j.firesaf.2017.12.002

6. Sturk, D. H. (2015). Fire Tests on E-vehicle Battery Cells and Packs. Traffic injury prevention, vol. 16 Suppl 1, 159-164

7. FSV. (2015). RVS 09.03.11 Tunnel Risikoanalysemodell. Wien: Forschungsgesellschaft Straße, Schiene, Verkehr.

Hinweis des Verlags. Der Verlag bleibt in Hinblick auf geografische Zuordnungen und Gebietsbezeichnungen in veröffentlichten Karten und Institutsadressen neutral. 